\title{
EXTENDING CONGRUENCES ON SEMIGROUPS
}

\author{
BY \\ A. R. STRALKA
}

\begin{abstract}
The two main results are: (1) Let $S$ be a semigroup which satisfies the relation $a b c d=a c b d$, let $A$ be a subsemigroup of $\operatorname{Reg} S$ which is a band of groups and let $[\varphi]$ be a congruence on $A$. Then $[\varphi]$ can be extended to a congruence on $S$. (2) Let $S$ be a compact topological semigroup which satisfies the relation $a b c d=a c b d$, let $A$ be a closed subsemigroup of Reg $S$ and let $[\varphi]$ be a closed congruence on $A$ such that $\operatorname{dim} \varphi(A) / \mathscr{H}=0$. Then $[\varphi]$ can be extended to a closed congruence on $S$.
\end{abstract}

For a semigroup $S$ let us define $\operatorname{Reg}(S)$ to be the set of those elements $x$ of $S$ for which there is an element $y \in S$ such that $x y x=x$ and $y x y=y$. In this paper we consider the following question: Let $S$ be a (compact topological) semigroup, let $A$ be a (closed) subsemigroup of $\operatorname{Reg}(S)$ and let $[\varphi]$ be a (closed) congruence on $A$. When can $[\varphi]$ be extended to a (closed) congruence on $S$ ? That is, when is there a (closed) congruence [ $\Phi]$ on $S$ such that [ $\Phi] \cap A \times A=[\varphi]$ ? It is known (cf. [6]) that any congruence on any sublattice of distributive lattice $L$ can be extended to $L$. In fact this property, known as the congruence extension property, serves to characterize distributive lattices. The topological analog of this result for compact topological lattices of finite breadth was proved in [13]. In the same paper an example was given of a compact distributive topological lattice of infinite breadth which does not share this property.

In [16] Wallace, in a result which he attributes to Borsuk, gives conditions under which, given a closed congruence $[\varphi]$ on a closed subsemigroup $A$ of a compact semigroup $S,[\varphi] \cup \Delta_{S \times S}$ is a closed congruence on $S$. Further results of this nature were established by Borrego in [3]. In [12] the author showed implicitly that if $S$ is a compact topological semigroup and $[\varphi]$ is a closed congruence on $E(S)$, the set of idempotents of $S$, such that $\operatorname{dim} \varphi(E(S))=0$ then $[\varphi]$ can be extended to a closed congruence on $S$.

Our main result for nontopological semigroups is that if $S$ is a semigroup which satisfies the relation $a b c d=a c b d$ then any congruence on any subsemigroup $A$ of $\operatorname{Reg} S$ where $A$ is a band of groups can be extended to a congruence on $S$. For topological semigroups we obtain the result that if $S$ is a compact topological semigroup which satisfies the relation $a b c d=a c b d, A$ is a closed subsemigroup of $\operatorname{Reg}(S)$ and $[\varphi]$ a closed congruence on $A$ such that $\operatorname{dim} \varphi(A) / \mathscr{H}=0$, then $[\varphi]$

Received by the editors February 12, 1971.

AMS 1969 subject classifications. Primary 2205, 2092, 2093.

Key words and phrases. Topological semigroup, semigroup, congruence, naturally ordered band, $N$-inversive.

Copyright (C) 1972, American Mathematical Society 
can be extended to a closed congruence on $S$. Finally, in $\S 6$ we apply some of these results to obtain representations of compact semigroups by $H L$-semigroups. This sort of representation was first discussed by Clark and Carruth in [4].

We should note that at many points we touch upon the concepts developed by M. Yamada in [14] and [15].

1. Definitions and notation. We shall assume that all topological spaces are Hausdorff. Congruences will be denoted by $[\varphi]$ where $\varphi$ is the canonical homomorphism associated with $[\varphi]$. For a semigroup $S$ by $S^{0}$ we shall mean the semigroup $S$ if $S$ has a zero and $S \cup\{0\}$ if $S$ does not have a zero. The quasi-orders associated with the Green equivalences will be denoted in the following fashion: $x \leqq y(\mathscr{L})$ if and only if $S x \cup\{x\} \subseteq S y \cup\{y\}$. < will mean strict inequality. We shall use the same notation regarding the Green relations as [5]. For an element $x$ of a semigroup $S$ we define $\tau(x)$ to be $E(S) \cap H_{x}$ if it is nonempty. Recall that a band is an idempotent semigroup. For a compact topological semigroup $S, M(S)$ will denote its minimal ideal. $X^{*}$ will denote the topological closure of the space $X$.

2. Extending congruences on naturally ordered bands. On a band there is a partial order $(\mathscr{A})$ defined by

$$
e \leqq f(\mathscr{A}) \text { if and only if } e f=f e=e .
$$

In [7] J. M. Howie defines a band to be naturally ordered if its multiplication is compatible with $\mathscr{A}$, i.e., if $e \leqq f(\mathscr{A})$ and $g \leqq h(\mathscr{A})$, then $e g \leqq f h(\mathscr{A})$. It has been noted by L. W. Anderson and R. P. Hunter in [2] that a band is naturally ordered if and only if it satisfies the relation $a b c a=a c b a$. While in [14] M. Yamada proved that a band satisfies the relation $a b c d=a c b d$ if and only if it satisfies the relation $a b c a=a c b a$. Bands which satisfy the relation $a b c d=a c b d$ were called normal bands by M. Yamada and N. Kimura [8]. In this paper we shall use the term naturally ordered band rather than normal band and reserve the term normal for use in another context.

In this section we prove that naturally ordered bands have the congruence extension property.

LEMMA 2.1. Let $S$ be a naturally ordered band and let $A$ be a subsemigroup of $S$ which satisfies the condition

$$
\text { if } s \in S \text { then there is } a \in A \text { such that asa }=a .
$$

If a relation $[\theta]$ is defined on $S$ as follows:

$$
(x, y) \in[\theta] \text { if and only if there is } a \in A \text { such that } x a x=y a y
$$

then $[\theta]$ is a congruence on $S$ such that $\theta(A)$ is the maximal rectangular band homomorphic image of $A$. 
Proof. It is readily apparent that $[\theta]$ is reflexive and symmetric. Suppose that $(x, y),(y, z) \in[\theta]$. There are $a, b \in A$ such that $x a x=y a y$ and $y b y=z b z$. Then

$$
\begin{aligned}
x a b x & =(x a x) b(x a x)=(y a y) b(y a y) \\
& =(y b y) a(y b y)=(z b z) a(z b z)=z a b z .
\end{aligned}
$$

Hence $[\theta]$ is an equivalence relation on $S$. That $[\theta]$ is compatible follows from the fact that if $(x, y) \in[\theta]$ with $x a x=y a y$ for some $a \in A$ then for $r \in S$,

$$
(r x) a(r x)=r(x a x) r(x a x)=r(y a y) r(y a y)=(r y) a(r y) .
$$

Thus $[\theta]$ is a congruence on $S$.

Let $\bar{x}, \bar{y} \in \theta(S)$. Choose $x \in \theta^{-1}(\bar{x})$ and $y \in \theta^{-1}(\bar{y})$. By $\left(^{*}\right)$ there is $a \in A$ such that $a y a=a$. Then $x a x=x a y a x=(x y x) a(x y x)$. Thus

$$
\bar{x}=\theta(x)=\theta(x y x)=\bar{x} \bar{y} \bar{x} .
$$

Hence $\theta(S)$ is a rectangular band. Now suppose that $[\rho]$ is a congruence on $A$ such that $\rho(A)$ is a rectangular band. Let $(x, y) \in[\theta] \cap A \times A$. There is $a \in A$ such that $x a x=y a y$. Then since $\rho(A)$ is a rectangular band we must have

$$
\rho(x)=\rho(x) \rho(a) \rho(x)=\rho(x a x)=\rho(y a y)=\rho(y) \rho(a) \rho(y)=\rho(y) .
$$

Hence $[\theta] \cap A \times A \subseteq[\rho]$.

The next lemma is well known.

LEMMA 2.2. Let $S$ be a rectangular band and let $A$ be a subsemigroup of $S$. Then every congruence on $A$ can be extended to $S$.

We now show that naturally ordered bands have the congruence extension property. In fact, we prove a somewhat stronger result.

THEOREM 2.1. Let $S$ be a naturally ordered band, let $A$ be a subsemigroup of $S$ and let $[\psi]$ be a congruence on $A$. Then $[\psi]$ can be extended to a congruence $[\Psi]$ on $S$ which has the property that given $a \in A$ and $x \in S$ with $\Psi(a)=\Psi(x)$ there is $b \in A$ such that $\Psi(b)=\Psi(x)$ and $b x b=b$.

Proof. Let $\left\{D_{\alpha} ; \alpha \in \Omega\right\}$ be the collection of $\mathscr{D}$-classes of $\psi(A)$. From each $D_{\alpha}$ select an element $b_{\alpha}$ to form a set $B=\left\{b_{\alpha} ; \alpha \in \Omega\right\}$. Since $\psi(A)$ is a naturally ordered band for each $\alpha \in \Omega$ the map $x \rightarrow x b_{\alpha} x$ is a homomorphism of $\psi(A)$ onto $\psi(A) b_{\alpha} \psi(A)$. Then since $N_{\alpha}=\psi(A) b_{\alpha} \psi(A) \backslash D_{\alpha}$ is an ideal of $\psi(A) b_{\alpha} \psi(A)$, the Reesquotient $\psi(A) b_{\alpha} \psi(A) / N_{\alpha}$ can be formed. Let $\psi_{\alpha}$ be the natural homomorphism of $\psi(A)$ onto $\psi(A) b_{\alpha} \psi(A) / N_{\alpha}$. Note that $\psi_{\alpha}(\psi(A))$ is isomorphic with either $D_{\alpha}$ or $D_{\alpha}^{0}$. Also the collection of congruences $\left\{\left[\psi_{a}\right] ; \alpha \in \Gamma\right\}$ separates points of $\psi(A)$. This implies that $[\psi]=\bigcap\left\{\left[\psi_{a} \circ \psi\right] ; \alpha \in \Gamma\right\}$.

Next, for $\alpha \in \Omega$ we define the sets $W_{\alpha}=\left(\psi_{\alpha} \circ \psi\right)^{-1}\left(D_{\alpha}\right), X_{\alpha}=\left\{s \in S\right.$; if $w \in W_{\alpha}$ then $w s w \neq w\}$ and $S_{\alpha}=S \backslash X_{\alpha}$. Let $\alpha \in \Omega$ be fixed. It will entail no loss of generality to assume that $W_{\alpha} \neq \varnothing$. We claim that $X_{\alpha}$ is a prime ideal of $S$. Let $x \in X_{\alpha}$ and let 
$s \in S$. If there is $w \in W_{\alpha}$ such that $w=w s x w$ then because $S$ is naturally ordered $w=w s x w=(w s w)(w x w)$. Because $S$ is a band this implies that $w s w=w=w x w$. But this contradicts our assumption that $x \in X_{\alpha}$. Now suppose that $s, t \in S \backslash X_{\alpha}$. There are $w, w^{\prime} \in W_{\alpha}$ such that $w s w=w$ and $w t w^{\prime}=w^{\prime}$. Then, since $W_{\alpha}$ is a semigroup $w w^{\prime} \in W_{\alpha}$ and because $S$ is naturally ordered,

$$
w w^{\prime} s t w w^{\prime}=(w s w)\left(w^{\prime} t w^{\prime}\right)=w w^{\prime} .
$$

Thus $X_{\alpha}$ is a prime ideal of $S$. As a consequence of this fact $S_{\alpha}$ is a naturally ordered band. On $S_{\alpha}$ we define a relation $\left[\theta_{\alpha}\right]$ by

$$
(x, y) \in\left[\theta_{\alpha}\right] \text { if and only if there is } w \in W_{\alpha} \text { such that } x w x=y w y .
$$

Then because as a subsemigroup of $S_{\alpha}, W_{\alpha}$ satisfies the condition (*) of Lemma $2.1,\left[\theta_{\alpha}\right]$ is a congruence on $S_{\alpha}$ such that $\theta_{\alpha}\left(S_{\alpha}\right)$ is a rectangular band and $\theta_{\alpha}\left(W_{\alpha}\right)$ is the maximal rectangular band homomorphic image of $W_{\alpha}$. From the last named property of $\theta_{\alpha}$ it follows that there must be a homomorphism $\gamma_{\alpha}$ mapping $\theta_{\alpha}\left(W_{\alpha}\right)$ onto $\psi_{\alpha} \circ \psi\left(W_{\alpha}\right) . \theta_{\alpha}\left(S_{\alpha}\right)$ is a rectangular band so from Lemma $2.2\left[\gamma_{\alpha}\right]$ can be extended to a congruence $\left[\Gamma_{\alpha}\right]$ on $\theta_{\alpha}\left(S_{\alpha}\right)$. We summarize with the following commutative diagram

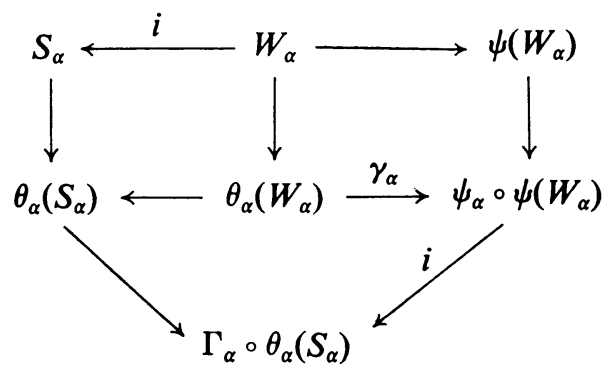

where in each case $i$ is the appropriate inclusion map. Next, we extend [ $\Gamma_{\alpha} \circ \theta_{\alpha}$ ] from $S_{\alpha}$ to $S$. If $X_{\alpha}=\varnothing$ then $S_{\alpha}=S$ and we define $\left[\Psi_{\alpha}\right]=\left[\Gamma_{\alpha} \circ \theta_{\alpha}\right]$. However, if $X_{\alpha} \neq \varnothing$ we define

$$
\left[\Psi_{\alpha}\right]=\left[\Gamma_{\alpha} \circ \theta_{\alpha}\right] \cup\left(X_{\alpha} \times X_{\alpha}\right)
$$

Obviously, in either case $\left[\Psi_{\alpha}\right]$ is a congruence on $S$ and $\Psi_{\alpha}(S)$ is a rectangular band in the first case and a rectangular band with zero appended in the second

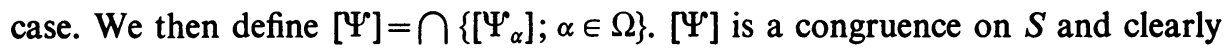
$[\Psi]$ is an extension of $[\psi]$.

Now let $a \in A$ and $x \in S$ with $(a, x) \in[\Psi]$. Choose $\alpha \in \Omega$ so that $\psi(a) \in D_{\alpha}$. Then it follows that $(a, x) \in\left[\Psi_{\alpha}\right] . W_{\alpha}$ satisfies condition $\left(^{*}\right)$ of Lemma 2.1 as a subsemigroup of $S_{\alpha}$ so there is $w^{\prime} \in W_{\alpha}$ such that $w^{\prime} x w^{\prime}=w^{\prime}$. Let $w=a w^{\prime} a$. Then $w \in W_{\alpha}$ and $w a w=w=w x w$. Since $w \in W_{\alpha}$ we have $\psi(w) b_{\alpha} \psi(w) \in D_{\alpha}$. This implies that $\psi(w)=\psi(w) \psi(a) \psi(w) \in D_{\alpha}$. Because $w=a w a$ it then follows that $\psi(w)=\psi(a)$. This completes our proof. 
3. Extending congruences on bands of groups. Let $S$ be a band of groups such that $E(S)$ is a subsemigroup of $S$. The map $\tau: S \rightarrow E(S)$ defined by $\tau(x)$ $=H_{x} \cap E(S)$ is a homomorphism. A fortiori every congruence on $E(S)$ can be extended to $S$. However, it will be useful to determine for any given congruence on $E(S)$ its minimal extension to $S$. This will be particularly useful in $\$ 4$. The congruence [ $\Phi]$ on $S$ is said to be the minimal extension of the congruence $[\varphi]$ on $E(S)$ if given any congruence $[\theta]$ on $S$ such that $[\theta]$ is an extension of $[\varphi]$ then $[\Phi] \subseteq[\theta]$.

THEOREM 3.1. Let $S$ be a band of groups such that $E(S)$ is a subsemigroup of $S$ and let $[\varphi]$ be a congruence on $E(S)$. For $x \in S$ define $\sigma(x)=\varphi^{-1}(\varphi(\tau(x)))$. Then the

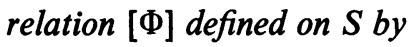

$$
(x, y) \in[\Phi] \text { if and only if } \sigma(x) x \sigma(x) \cap \sigma(y) y \sigma(y) \neq \varnothing
$$

is the minimal extension of $[\varphi]$ to $S$. Moreover, if $e \in E(S)$ has the property

$$
\text { if } f \in E(S) \text { and } \varphi(e)=\varphi(f) \text { then } e f e=e,
$$

then $\Phi$ restricted to $H_{e}$ is an isomorphism. Also if $S$ is a compact topological semigroup and $[\varphi]$ is a closed congruence on $E(S)$ then $[\Phi]$ is a closed congruence on $S$.

Before we prove our theorem it is necessary to establish the following lemma. It is assumed that we are operating under the hypotheses as stated above.

LEMMA 3.1. (1) If $a b \in H_{e}$ and $e \in E(S)$ then $a b=e a e b e$.

(2) If $e, f \in \sigma(x)$ and $g=\tau(e x f)$ then $g \in \sigma(x)$ and $g x g=e x f$.

(3) If $(x, y) \in[\Phi]$ then $\sigma(x)=\sigma(y)$.

(4) If efxef $\in D_{\text {ef }}$ then efxef $=$ efexef $=e f x f e f$.

Proof. (1) If $a b \in H_{e}$ then $a b=e a b e$. Then, since $e a \in R_{e}$ and $b e \in L_{e}$, we have

$$
a b=(e a)(b e)=(e a) \tau(e a) \tau(b e)(b e)=e a e b e .
$$

(2) Since $\tau$ is a homomorphism of $S$ onto $E(S) g=\tau(e x f)=\tau(e) \tau(x) \tau(f)=e \tau(x) f$. Since $e, f, \tau(x) \in \sigma(x)$ and $\sigma(x)$ is a semigroup $g \in \sigma(x)$. Then from (1)

$$
\operatorname{exf}=g(\text { exf }) g=(g e g) x(g f g)=g x g \text {. }
$$

(3) From (2) there is $g \in \sigma(x) \cap \sigma(y)$ such that $g x g=g y g$. Then we have $\sigma(x)$ $=\varphi^{-1}(\varphi(g))=\sigma(y)$.

(4) Let $\theta$ be the natural homomorphism of $S$ onto $S / \mathscr{D}$. Then since $S / \mathscr{D}$ is abelian and efxef $\in D_{e f}$,

$$
\theta(e f x e f)=\theta(e f)=\theta(\text { efexef })=\theta(e f x f e f) .
$$

From (1) above we obtain

$$
\text { efexef }=(e f e)(e f)(x e f)=e f x e f=(e f x)(e f)(f e f)=e f x f e f .
$$

This completes the proof of our lemma. 
Proof of Theorem 3.1. It is apparent that $[\Phi]$ is a reflexive and symmetric relation on $S$. Suppose that $(x, y),(y, z) \in[\Phi]$. From Lemma 3.1 it follows that $\sigma(x)=\sigma(y)=\sigma(z)$. Lemma 3.1 also yields the fact that there are $e, f \in \sigma(x)$ such that $e x e=e y e$ and $f y f=f z f$. Then $e f x e f$, efyef, efzef $\in H_{e f}$ and by part (4) of Lemma 3.1

$$
\begin{aligned}
\text { efxef } & =\text { efexef }=\text { efeyef } \\
& =\text { efyef }=\text { efyfef } \\
& =\text { efzfef }=\text { efzef. }
\end{aligned}
$$

Hence, $(x, z) \in[\Phi]$ and $[\Phi]$ is an equivalence relation on $S$.

Now let $(x, y) \in[\Phi]$ and let $r \in S$. There is $g \in \sigma(x)=\sigma(y)$ such that $g x g=g y g$. Then it is the case that $\tau(r g) r x \tau(r g), \tau(r g) r y \tau(r g) \in H_{\tau(r g)}$. Using the facts that $\tau$ is a homomorphism and part (4) of Lemma 3.1 we obtain

$$
\begin{aligned}
\tau(r g) r x \tau(r g) & =\tau(r g) r \tau(r) g x \tau(r g)=\tau(r g) r \tau(r) g x g \tau(r g) \\
& =\tau(r g) r \tau(r) g y g \tau(r g)=\tau(r g) r y \tau(r g) .
\end{aligned}
$$

Then since $\sigma(r) \sigma(x) \subseteq \sigma(r x)$ we conclude that $(r x, r y) \in[\Phi]$. Thus it will follow that $[\Phi]$ is a congruence on $S$.

Suppose that $[\Psi]$ is any extension of $[\varphi]$ to $S$. Let $(x, y) \in[\Phi]$. From part (2) of Lemma 3.1 there is $g \in E(S)$ such that $g x g=g y g$ and $\varphi(g)=\varphi(\tau(x))=\varphi(\tau(y))$. Since $[\Psi]$ is also an extension of $[\varphi]$ it follows that $(\tau(x) x \tau(x), g x g),(\tau(y) y \tau(y), g y g)$ $\in[\Psi]$. By transitivity we have $(x, y) \in[\Psi]$. Therefore $[\Phi] \subseteq[\Psi]$. Thus $[\Phi]$ is the minimal extension of $[\varphi]$ to $S$.

Now suppose that $e$ is a member of $E(S)$ which satisfies the condition $\left(^{* *}\right)$ above. Let $(x, y) \in H_{e}$ and suppose that $(x, y) \in[\Phi]$. There is $g \in \sigma(x)=\sigma(y)$ such that $g x g=g y g$. Since $e$ satisfies $\left({ }^{* *}\right) e g e=e$ and $g y g \in D_{e}$. Then by parts (1) and (4) of Lemma 3.1. we obtain

$$
x=\text { exe }=\text { egexege }=\text { egxge }=\text { egyge }=\text { egeyege }=\text { eye }=y .
$$

Thus $\Phi$ restricted to $H_{e}$ is an isomorphism.

Suppose that $S$ is a compact topological semigroup and $[\varphi]$ is a closed congruence on $E(S)$. Then $\tau$ is a continuous homomorphism on $S$. Let $\left\{\left(x_{\alpha}, y_{\alpha}\right) ; \alpha \in \Gamma\right\}$ be a net in [Ф] converging to $\left(x_{0}, y_{0}\right)$. Then for each $\alpha \in \Gamma$ there is $g_{\alpha} \in \sigma\left(x_{\alpha}\right)=\sigma\left(y_{\alpha}\right)$ such that $g_{\alpha} x_{\alpha} g_{\alpha}=g_{\alpha} y_{\alpha} g_{\alpha}$. There is a subnet $\left\{\left(x_{\alpha}, y_{\alpha}\right) ; \alpha \in \Gamma^{\prime}\right\}$ of $\left\{\left(x_{\alpha}, y_{\alpha}\right) ; \alpha \in \Gamma\right\}$ which converges to $\left(x_{0}, y_{0}\right)$ such that the net $\left\{g_{\alpha} ; \alpha \in \Gamma^{\prime}\right\}$ converges to some point $g$. Since $E(S)$ is closed $g \in E(S)$. Since $\tau$ is continuous $\left\{\tau\left(x_{\alpha}\right) ; \alpha \in \Gamma\right\}$ must converge to $\tau\left(x_{0}\right)$ and since $\varphi$ is continuous it follows that $g=\varphi\left(x_{0}\right)$. Then by continuity of multiplication we have $g x_{0} g=g y_{0} g$. Therefore $\left(x_{0}, y_{0}\right) \in[\Phi]$. Hence $[\Phi]$ is a closed congruence.

4. Extending congruences on semigroups which satisfy the relation $a b c d=a c b d$. For a semigroup $S$ recall that $\operatorname{Reg}(S)=\{x \in S$; there is $y \in S$ such that $x y x=x$ and $y x y=y\}$. In [14] Yamada defines a semigroup to be $N$-inversive if it is regular and satisfies the relation $a b c d=a c b d$. He then proved that such a semigroup must 
be a band of groups whose set of idempotents is a naturally ordered band. If $S$ is a semigroup which satisfies the relation $a b c d=a c b d$ then it is easily proved that $\operatorname{Reg}(S)$ is a subsemigroup of $S$ such that $E(S)=E(\operatorname{Reg}(S))$. Since $\operatorname{Reg}(S)$ must also satisfy the relation $a b c d=a c b d$ it must be an $N$-inversive semigroup.

LeMma 4.1. Let $S$ be a semigroup which satisfies the relation abcd=acbd. Then there is a homomorphism $\Omega$ of $S$ into an $N$-inversive semigroup such that $\Omega$ restricted to $\operatorname{Reg}(S)$ is an isomorphism.

Proof. We first prove two preliminary facts. For $e \in E(S)$ we define $N(e)=\{x \in S ;$ exe $<e(\mathscr{D})\}$. Then

(i) If $e \in E(S)$ then $N(e)$ is a prime ideal of $S$.

Suppose that $e \in E(S), x \in N(e), r \in S$ and $r x \notin N(e)$. Then erxe $\in H_{e}$. Since $H_{e}$ is a group there is $h \in H_{e}$ such that $h($ erxe $)=(h r)(x e)=e$. This implies that $h r \in R_{e}$ and $x e \in L_{e}$. From Theorem 2.17 of [5] we have $E(S) \cap L_{h r} \cap R_{x e} \neq \varnothing$. Let $f$ be the idempotent of $L_{h r} \cap R_{x e}$. Then $(x e)(h r)=x h r \in H_{f}$. Since $H_{f}$ is a group there is $g \in H_{f}$ such that $(x h r) g=f$. Then

$$
(x e)(h r g)(x e)=(x h r g)(x e)=f x e=x e .
$$

Thus $x e \in \operatorname{Reg}(S)$. As noted above $\operatorname{Reg}(S)$ is a band of groups so $E(S) \cap H_{x e} \neq \varnothing$. This implies that exe $\in H_{e}$ contrary to our assumption that $x \in N(e)$. Therefore $N(e)$ is an ideal of $S$.

Let $x, y \in S \backslash N(e)$. exe, eye $\in H_{e}, H_{e}$ is a group and $S$ satisfies the relation abcd $=a c b d$ so it follows that exye $=($ exe $)(e y e) \in H_{e}$. Hence $N(e)$ is a prime ideal of $S$.

(ii) If $e \in E(S)$ then $J_{e}=D_{e}$.

Recall that $J_{e}$ is the set of those elements of $S$ which generate the same principal ideal as $e$. Let $x \in J_{e}$. Since $e \in E(S)$ we may assume that $x=s e t$ for some $s, t \in S$. From (i) above $x \notin N(e)$ so exe $\in H_{e}$. This implies that ese, ete $\in H_{e}$. Hence se $\in L_{e}$. Then we have $(e, s e) \in \mathscr{L}$ and (se, set $) \in \mathscr{R}$. Thus $(e, x) \in \mathscr{D}$, i.e. $J_{e}=D_{e}$. Thus (ii) is proved.

Let $e \in E(S)$. Since $E(S) \cap D_{e}$ is a semigroup the map $\tau$ restricted to $D_{e}$ is a homomorphism of $D_{e}$ onto $D_{e} \cap E(S)$. We define a map $\omega_{e}: S \rightarrow D_{e}^{0}$ by

$$
\begin{aligned}
\omega_{e}(x) & =\tau(x e x) x \tau(x e x) & & \text { if } x \in S \backslash N(e), \\
& =0 & & \text { if } x \in N(e) .
\end{aligned}
$$

Clearly, $\omega_{e}$ is the identity map when restricted to $D_{e}$. As a consequence $D_{e}$ is contained in the range of $\omega_{e}$. For $x, y \in S \backslash N(e)$ we have

$$
\begin{aligned}
\omega_{e}(x y) & =\tau(\text { xyexy }) x y \tau(\text { xyexy }) \\
& =\tau(\text { xexyey }) x y \tau(\text { xexyey })=\tau(\text { xex }) \tau(\text { yey }) x y \tau(\text { xex }) \tau(\text { yey }) \\
& =(\tau(\text { xex }) x \tau(\text { xex }))(\tau(\text { yey }) y \tau(\text { yey }))=\omega_{e}(x) \omega_{e}(y) .
\end{aligned}
$$

Then since $N(e)$ is a prime ideal of $S$ it follows that $\omega_{e}$ is a homomorphism of $S$ into $D_{e}^{0}$. Form a subset $F$ of $E(S)$ by selecting one and only one idempotent from 
each regular $\mathscr{D}$-class of $S$. Then $\Omega: S \rightarrow \prod\left\{D_{f}^{0}, f \in F\right\}$ defined by $\Omega(s)_{e}=\omega_{e}(s)$ is a homomorphism of $S$ into the $N$-inversive semigroup $\prod \psi\left\{D_{f}^{0} ; f \in F\right\}$. It then follows easily that $\Omega$ restricted to $\operatorname{Reg}(S)$ is an isomorphism.

Lemma 4.2. Let $S$ be an $N$-inversive semigroup; let $\varphi$ be a homomorphism of $S$ onto the semigroup $T$ such that $\varphi$ restricted to $E(S)$ is an isomorphism; and let $K=\varphi^{-1}(E(T))$. Then the relation $[\xi]$ defined by

$$
(x, y) \in[\xi] \text { if and only if } K x K=K y K
$$

is a congruence on $S$ and $[\varphi]=[\xi] \cap \mathscr{H}$.

Proof. Clearly, $[\xi]$ is an equivalence relation on $S$. Let $(s, t) \in[\xi]$; let $r \in S$; and let $k_{1}, k_{2} \in K$. Then there are $k_{3}, k_{4} \in K$ such that $k_{1} s k_{2}=k_{3} t k_{4} . K$ is a band of groups so $\tau\left(k_{1}\right)$ exists. Hence

$$
\begin{aligned}
k_{1} r s k_{2} & =\tau\left(k_{1}\right) k_{1} r s k_{2}=\tau\left(k_{1}\right) r k_{1} s k_{2} \\
& =\tau\left(k_{1}\right) r k_{3} t k_{4}=\tau\left(k_{1}\right) k_{3} r t k_{4} .
\end{aligned}
$$

Hence $K r s K \subseteq K r t K$. It then follows by symmetry that [ $\xi]$ is a congruence on $S$. Then because $\mathscr{H}$ is a congruence so is $\mathscr{H} \cap[\xi]$.

Let $(x, y) \in[\varphi]$. Since $\varphi$ restricted to $E(S)$ is an isomorphism we have $(x, y) \in \mathscr{H}$. Let $e=\tau(x)=\tau(y) . \varphi$ restricted to $H_{e}$ is a group homomorphism so there are $k, k^{\prime} \in \varphi^{-1}(\varphi(e))$ such that $y=k x$ and $x=k^{\prime} y$. Hence

$$
K y K=K k x K \subseteq K x K=K k^{\prime} y K \subseteq K y K .
$$

Thus $(x, y) \in[\xi]$. Therefore $[\varphi] \subseteq \mathscr{H} \cap[\xi]$.

Now let $(x, y) \in \mathscr{H} \cap[\xi]$. There are $k, k^{\prime} \in K$ such that $y=k x k^{\prime}$. Let $f=\tau(\varphi(x))$ $=\tau(\varphi(y))$. Then

$$
\begin{aligned}
\varphi(y) & =f \varphi(y) f=f \varphi\left(k x k^{\prime}\right) f=f \varphi(k) \varphi(x) \varphi\left(k^{\prime}\right) f \\
& =(f \varphi(k) f) \varphi(x)\left(f \varphi\left(k^{\prime}\right) f\right)=f \varphi(x) f=\varphi(x) .
\end{aligned}
$$

THEOREM 4.1. Let $S$ be a semigroup which satisfies the relation abcd=acbd and let $A$ be a subsemigroup of $\operatorname{Reg}(S)$ such that $A$ is a band of groups. Then every congruence on $A$ can be extended to $S$.

Proof. As a consequence of Lemma 4.1 we may assume that $S$ is an $N$-inversive semigroup. Let $[\rho]$ be any congruence on $A$ and let $[\psi]=[\rho] \cap(E(A) \times E(A))$. From Theorem 3.1 there is a minimal congruence on $A$ which is an extension of $[\psi]$. Denote this congruence by $[\Psi]$. Let $[\zeta]$ be the extension of $[\psi]$ to $E(S)$ obtained in the same manner as that used in the proof of Theorem 2.1. From Theorem 3.1,

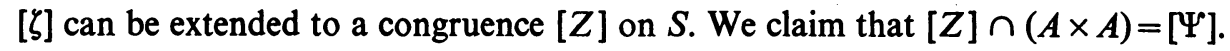
Let $(x, y) \in[Z] \cap(A \times A)$. Then $\sigma(x) x \sigma(x) \cap \sigma(y) y \sigma(y) \neq \varnothing$ where $\sigma(x)=\zeta^{-1}(\zeta(\tau(x)))$. From Lemma $3.1 \sigma(x)=\sigma(y)$ and there are $e, f \in \sigma(x)$ such that $e x e=f y f$. Then from Theorem 2.1 there is $g \in E(A) \cap \sigma(x)$ such that exe $=f y f$. Then from Theorem 
2.1 there is $g \in E(A) \cap \sigma(x)$ such that $g e g=g f g=g$. With this fact we obtain

$$
g x g=(g e g) x(g e g)=g e x e g=g f y f g=(g f g) y(g f g)=g y g .
$$

Therefore $(x, y) \in[\Psi]$ and $[Z] \cap(A \times A) \subseteq[\Psi]$. Obviously, $[\Psi] \subseteq[Z] \cap(A \times A)$ so we have $[Z] \cap(A \times A)=[\Psi]$.

$[\Psi]$ is the minimal extension of $[\psi]$ to $A$ so it must be the case that $[Z] \cap(A \times A)$ $\subseteq[\rho]$. Let $\varphi$ be the canonical homomorphism from $Z(A)$ onto $\rho(A)$. Note that $\varphi$ is an isomorphism of $E(Z(A))$ onto $E(\rho(A))$. Let $K=\varphi^{-1}(E(\rho(A)))$. From Lemma 4.2 we know that $[\varphi]=[\xi] \cap \mathscr{H}$ where $[\xi]$ is defined on $A$ by

$$
(x, y) \in[\xi] \text { if and only if } K x K=K y K \text { for } x, y \in A .
$$

The same proof as that used in Lemma 4.2 shows that the relation [ $\Xi$ ] defined on $S$ by

$$
(x, y) \in[\Xi] \text { if and only if } K x K=K y K \text { for } x, y \in S
$$

is a congruence on $S$ and obviously $[\Xi]$ is an extension of $[\xi]$ to $S$. It then follows immediately that $[\Xi] \cap \mathscr{H}$ is an extension of $[\rho]$ to $S$.

5. Extending congruences on compact semigroups. We begin by giving two examples which show that results directly analogous to Theorem 2.1 and Theorem 4.1 are not possible for compact topological semigroups. Our first example also appears in [13].

Example 5.1. Let 2 denote the semigroup $\{0,1\}$ where 0 is a zero and 1 is the identity element. Let $X$ be the Cartesian product of a countable collection of copies of 2 endowed with the Cartesian product topology and coordinatewise operations. $X$ is then a compact zero-dimensional topological semilattice. It can easily be shown that $X$ contains a closed subsemigroup $S$ which is a chain (i.e., for $s, t \in S, s t \in\{s, t\})$ homeomorphic with the Cantor set. Let $\psi$ be the usual dimension-raising homomorphism of $S$ onto $I$. It was proved in [13] that $X$ does not have any dimension-raising homeomorphisms. Hence it follows that $[\psi]$ cannot be extended to a closed congruence on $X$.

Example 5.2. In $E^{2}$ we define $D_{1}=\{(1 / 2,1)\}$. Then after having defined $D_{k}$ for all those positive integers $k$ such that $1 \leqq k \leqq i$ we define $D_{i+1}=\left\{\left(x \pm 1 / 3^{i}, 1 /(i+1)\right)\right.$; $\left.(x, 1 / i) \in D_{i}\right\}$. Let $S=\left(\bigcup_{i=1}^{\infty} D_{i}\right)^{*}$ and let $D_{\infty}=S \backslash \bigcup_{i=1}^{\infty} D_{i}$. Note that $D_{\infty}$ is the usual Cantor ternary set on the closed interval from $(0,0)$ to $(1,0)$. For $\infty \geqq m \geqq n$ $\geqq 1$ we define a map $\pi_{m n}: D_{m} \rightarrow D_{n}$ by letting $\pi_{m n}(x)$ be that point of $D_{n}$ which is nearest to $x$. We shall now use this collection of maps to define a multiplication on $S$. Let $x, y \in S$. There are $m, n \in Z^{+} \cup\{\infty\}$ such that $x \in D_{m}$ and $y \in D_{n}$. Let $k=\min \{m, n\}$. Then $x y$ is defined to be $\pi_{n k}(y)$. It follows that $S$ with this multiplication becomes a compact, naturally ordered topological band such that the $\mathscr{D}$-classes of $S$ are the $D_{i}$ 's defined above and $S=S D_{\infty} S=D_{\infty} S D_{\infty}$.

Now let $\psi$ be the usual dimension-raising map of $D_{\infty}$ onto $[(0,0),(1,0)]$. Since $D_{\infty}$ is right singular $[\psi]$ is a closed congruence on $D_{\infty}$. Suppose that $[\Psi]$ is a 
closed congruence on $S$ which is an extension of [ $\psi]$ ]. It follows that if $x, y \in D_{i}$ for $i \neq \infty$ then $(x, y) \in[\Psi]$. But this would imply that $[\Psi]=\mathscr{D}$. Hence $[\psi]$ cannot be extended to $S$.

Let 4 denote the four element rectangular band which is neither left singular nor right singular. As a consequence of Theorem 1 of [11] and its own natural order it follows that if $S$ is a compact zero-dimensional naturally-ordered band then the collection of continuous homomorphisms of $S$ into $4^{\circ}$, Hom $\left(S, 4^{\circ}\right)$, separates points.

In light of Examples 5.1 and 5.2 it is not unnatural that in the following theorem we include a condition which precludes the possibility of attempting to extend a dimension-raising homomorphism.

THEOREM 5.1. Let $S$ be a compact topological semigroup which satisfies the relation $a b c d=a c b d$ and let $A$ be a closed subsemigroup of $\operatorname{Reg} S$. If $[\psi]$ is a closed congruence on $A$ such that $\operatorname{dim} \psi(A) / \mathscr{H}=0$ then $[\psi]$ can be extended to a closed congruence on $S$.

Proof. As noted in $\$ 4, \operatorname{Reg} S$ is a band of groups. Then because $A$ is a closed subsemigroup of $\operatorname{Reg} S$ it must also be a band of groups.

Let $[\varphi]$ denote the restriction of $[\psi]$ to $E(A) . \varphi(E(A))$ is isomorphic with $\psi(A) / \mathscr{H}$ and $\operatorname{dim}(\psi(A) / \mathscr{H})=0$ so $\operatorname{dim} \varphi(E(A))=0$. Then as noted above Hom $\left(\varphi(E(A)), 4^{\circ}\right)$ separates points. Hence there is a family $\left\{\varphi_{\alpha} ; \alpha \in \Gamma\right\} \subseteq \operatorname{Hom}\left(E(A), 4^{\circ}\right)$ such that $[\varphi]=\bigcap\left\{\left[\varphi_{\alpha}\right] ; \alpha \in \Gamma\right\}$. We may assume that for all $\alpha \in \Gamma, \varphi_{\alpha}^{-1}(4) \neq \varnothing$. Then since $E(A)$ is compact we choose for each $\alpha \in \Gamma$ an element $e_{\alpha}$ of $M\left(\varphi_{\alpha}^{-1}(4)\right)$. Note that $M\left(\varphi_{\alpha}^{-1}(4)\right) \subseteq D_{e_{\alpha}}$. We define

$$
\begin{aligned}
& E_{\alpha 11}=\left\{e \in M\left(\varphi_{\alpha}^{-1}(4)\right) ; \varphi_{\alpha}(e)=\varphi_{\alpha}\left(e_{\alpha}\right)\right\}, \\
& E_{\alpha 12}=\left\{e \in M\left(\varphi_{\alpha}^{-1}(4)\right) ; \varphi_{\alpha}(e)=\varphi_{\alpha}\left(e_{\alpha} e\right) \neq \varphi_{\alpha}\left(e_{\alpha}\right)\right\}, \\
& E_{\alpha 21}=\left\{e \in M\left(\varphi_{\alpha}(4)\right) ; \varphi_{\alpha}(e)=\varphi_{\alpha}\left(e e_{\alpha}\right) \neq \varphi_{\alpha}\left(e_{\alpha}\right)\right\} .
\end{aligned}
$$

Then we define $N_{\alpha}$ to be the closed ideal of $S$ generated by $\left(e_{\alpha} S E_{\alpha 11} \cap e_{\alpha} S E_{\alpha 12}\right)$ $\cup\left(E_{\alpha 11} S e_{\alpha} \cap E_{\alpha 21} S e_{\alpha}\right) \cup \psi_{\alpha}^{-1}(0)$. It is easily seen that $N_{\alpha}$ does not meet $D_{e_{\alpha}}$. On $e_{\alpha} S$ we define a relation $\left[\lambda_{\alpha}\right]$ as follows

$$
\begin{aligned}
(x, y) \in\left[\lambda_{\alpha}\right] & \Leftrightarrow x=y, \text { or } \\
& \Leftrightarrow x, y \in N_{\alpha} \cap e_{\alpha} S, \text { or } \\
& \Leftrightarrow \text { there are } i \in\{1,2\} \text { and } e, f \in E_{\alpha 1 i} \cap e_{\alpha} S \text { such that } x e=y \text { and } y f=x .
\end{aligned}
$$

We claim that $\left[\lambda_{\alpha}\right]$ is a closed congruence on $e_{\alpha} S$. It is apparent that $\left[\lambda_{\alpha}\right]$ is both reflexive and symmetric. Suppose that $(x, y),(y, z) \in\left[\lambda_{\alpha}\right]$. There are $i, j \in\{1,2\}$ with $e, f \in E_{\alpha 1_{i}}$ and $g, h \in E_{\alpha 1 j}$ such that $x e=y, y f=x, y g=z$ and $z h=y$. If $i \neq j$ then $y e=y=y h$. Thus $y \in e_{\alpha} S E_{\alpha 11} \cap e_{\alpha} S E_{12}$. This implies that $x, y, z \in N_{\alpha}$. Hence $(x, z) \in\left[\lambda_{\alpha}\right]$. If $i=j$ then $f, g \in E_{\alpha 1 i}$ with $x g=x e g=z$ and $z f=z h f=x$. Therefore $(x, z) \in\left[\lambda_{\alpha}\right]$ so $\left[\lambda_{\alpha}\right]$ must be an equivalence relation on $e_{\alpha} S$. Now let $(x, y) \in\left[\lambda_{\alpha}\right]$ and let $r \in e_{\alpha} S$. There is $i \in\{1,2\}$ and $e, f \in E_{\alpha 1 i}$ such that $x e=y$ and $y f=x$. Obviously $r x e=r y$ and $r y f=r x$. Since $r \in e_{\alpha} S=e S$ we have $r=e r$. Hence $x r=x e r=y r$. 
Thus $\left[\lambda_{\alpha}\right]$ is a congruence on $e_{\alpha} S$. Let $\left\{\left(x_{\gamma}, y_{\gamma}\right) ; \gamma \in \Gamma\right\}$ be a net in $\left[\lambda_{\alpha}\right]$ converging to $(x, y)$. Then because the range of $\varphi_{\alpha}$ is finite $\left\{\left(x_{\gamma}, y_{\gamma}\right) ; \gamma \in \Gamma\right\}$ has a subnet $\left\{\left(x_{\gamma}, y_{\gamma}\right) ; \gamma \in \Gamma^{\prime}\right\}$ which converges to $(x, y)$ such that for each $\gamma \in \Gamma^{\prime}$ there is $e_{\gamma}, f_{\gamma} \in E_{\alpha 1 i}$ ( $i$ fixed) such that $x_{\gamma} e_{\gamma}=y_{\gamma}$ and $y_{\gamma} f_{\gamma}=x_{\gamma}$ and the nets $\left\{e_{\gamma} ; \gamma \in \Gamma^{\prime}\right\}$ and $\left\{f_{\gamma} ; \gamma \in \Gamma^{\prime}\right\}$ converge to $e$ and $f$ respectively. Since $E_{\alpha 1 i}$ is closed $e, f \in E_{\alpha 1 i}$. Then by the continuity of multiplication $x e=y$ and $y f=x$. Hence $\left[\lambda_{\alpha}\right]$ is a closed congruence on $e_{\alpha} S$. By the same reasoning as above it will follow that the relation $\left[\rho_{\alpha}\right]$ defined on $S e_{\alpha}$ by

$$
\begin{aligned}
(x, y) \in\left[\rho_{\alpha}\right] & \Leftrightarrow x=y, \text { or } \\
& \Leftrightarrow x, y \in N_{\alpha} \cap S e_{\alpha}, \text { or } \\
& \Leftrightarrow \text { there are } i \in\{1,2\} \text { and } e, f \in E_{\alpha i 1} \cap S e_{\alpha} \text { such that } e x=y \text { and } f y=x
\end{aligned}
$$

is a closed congruence on $S e_{\alpha}$. Since $S$ satisfies the relation $a b c d=a c b d$ the maps $x \rightarrow e_{\alpha} x$ and $x \rightarrow x e_{\alpha}$ are continuous homomorphisms of $S$ onto $e_{\alpha} S$ and $S e_{\alpha}$ respectively. Hence the relation $\left[\Phi_{\alpha}\right]$ defined by

$$
(x, y) \in\left[\Phi_{\alpha}\right] \text { if and only if }\left(e_{\alpha} x, e_{\alpha} y\right) \in\left[\lambda_{\alpha}\right] \text { and }\left(x e_{\alpha}, y e_{\alpha}\right) \in\left[\rho_{\alpha}\right]
$$

is a closed congruence on $S$. Moreover, it is easily seen that $\left[\Phi_{\alpha}\right]$ is an extension of $\left[\varphi_{\alpha}\right]$.

Let $[\Phi]=\bigcap\left\{\left[\Phi_{\alpha}\right] ; \alpha \in \Gamma\right\}$. Then $[\Phi]$ is a closed congruence on $S$ which is an extension of $[\varphi]$. We claim that $[\Phi] \cap A \times A \subseteq[\psi]$. Let $(x, y) \in[\Phi] \cap(A \times A)$. Then $\Phi(\tau(x))=\Phi(\tau(y))$. Let $e \in M\left(\varphi^{-1}(\varphi(\tau(x)))\right)$. There is a net $\left\{e_{\alpha} ; \alpha \in \Gamma\right\}$ (the $e_{\alpha}$ 's are the idempotents associated with the homomorphisms $\varphi_{\alpha}$ as above) which converges to some point $f \in E(A) \cap D_{e}$ such that for each $\alpha \in \Gamma, e_{\alpha} \leqq e(\mathscr{D})$. Since $(x, y) \in[\Phi],(x, y) \in\left[\Phi_{\alpha}\right]$ for all $\alpha \in \Gamma$. This implies that for each $\alpha \in \Gamma$ there is $g_{\alpha} \in M\left(\varphi_{\alpha}^{-1}(4)\right)$ such that $e_{\alpha} x g_{\alpha}=e_{\alpha} y$. After choosing appropriate subnets and then passing to the limit we obtain $g \in D_{e}$ such that $f x g=f y$. Then $e x e=e(f x g) e$ $=e(f y) e=e y e$. Hence

$$
\begin{aligned}
\psi(x) & =\psi(\tau(x) x \tau(x))=\psi(\tau(x)) \psi(x) \psi(\tau(x)) \\
& =\psi(e) \psi(x) \psi(e)=\psi(\text { exe })=\psi(\text { eye })=\psi(y)
\end{aligned}
$$

Therefore $[\Phi] \cap A \times A \Lambda[\psi]$.

Now, let $\gamma$ be the canonical homomorphism of $\Phi(A)$ onto $\psi(A)$ and let $K=\gamma^{-1}(\psi(E(A))) . K$ is a closed subsemigroup of $\Phi(S)$. As a consequence of Lemma 4.2 , the relation $[\eta]$ defined on $\Phi(S)$ by

$$
(x, y) \in[\eta] \text { if and only if } K x K=K y K \text { and }(x, y) \in \mathscr{H}
$$

is a congruence on $\Phi(S)$. It is then easily seen that $[\eta]$ is closed. It will then follow that $[\eta \circ \Phi]$ is the desired congruence.

For zero-dimensional semigroups we are able to prove a stronger result. Virtually the same proof which is used in Theorem 1.4 of [12] can be used for 
THEOREM 5.2. Let $S$ be a compact zero-dimensional topological semigroup which satisfies the relation abcd =acbd. Then $E(S)$ and $\operatorname{Reg}(S)$ are homomorphic retracts of $S$.

6. $H L$-semigroups. In [4] Clark and Carruth introduced the class of $H L$ semigroups. An $H L$-semigroup is a compact topological semigroup such that the Schützenberger group of each of its $\mathscr{H}$-classes is a Lie group. From [1] it follows that this property is equivalent to requiring that all maximal subgroups be Lie groups. Further work on $H L$-semigroups was done by Mislove in [10]. In this section we present several results which are complementary to those appearing in [4] and [10].

Let $S$ be a compact topological semigroup. $\mathscr{H} \mathscr{L}(S)$ is the collection of closed congruences on $S$ such that if $[\varphi] \in \mathscr{H} \mathscr{L}(S)$ then $\varphi(S)$ is an $H L$-semigroup. The general aim here as it had been in [4] and [10] is to determine classes $\mathscr{K}$ of compact semigroups such that if $S \in \mathscr{K}$ then $\mathscr{H} \mathscr{L}(S)$ separates points of $S$.

A normal semigroup is a semigroup having the property that $x S=S x$ for all $x \in S$. Recall that for such semigroups $\mathscr{D}$ reduces to $\mathscr{H}$ which is a congruence.

LEMMA 6.1. Let $S$ be a semigroup which is a band of groups such that $E(S)$ is a subsemigroup of $S$. Then the relation $[\eta]$ defined on $S$ by

$$
(x, y) \in[\eta] \quad \text { if and only if } E(S) x E(S)=E(S) y E(S)
$$

is a congruence on $S$ such that $\eta(S)$ is the maximal normal semigroup homomorphic image of $S$ and $\eta$ restricted to any $\mathscr{H}$-class of $S$ is an isomorphism. If in addition $S$ is a compact topological semigroup then $[\eta]$ is a closed congruence.

Proof. It is readily apparent that $[\eta]$ is an equivalence relation on $S$ and if $S$ is a compact semigroup then $[\eta]$ is closed. Also note that part (1) and the second half of part (2) of Lemma 3.1 will hold for $S$. Thus we have $(x, y) \in[\eta]$ if and only if $\tau(x) y \tau(x)=x$ and $\tau(y) x \tau(y)=y$. From this it follows that $\eta$ restricted to any $\mathscr{H}$-class of $S$ is an isomorphism.

Now suppose that $(x, y) \in[\eta]$ and $r \in S$. Let $g=\tau(r x)$. Then

$$
r x=g r x g=g r \tau(x) y \tau(x) g=\operatorname{grg} \tau(x) \operatorname{gyg} \tau(x) g=\operatorname{grgyg}=\operatorname{gryg} .
$$

This fact will lead to the conclusion that $[\eta]$ is a congruence on $S$. To see that $\eta(S)$ is the maximal normal homomorphic image of $S$ note that for $e \in E(S), \eta\left(D_{e}\right)$ is isomorphic with $\eta\left(H_{e}\right)$.

COROLlARY. Let $S$ be a semigroup which is a band of groups such that $E(S)$ is a subsemigroup of $S$. Then $\mathscr{H} \cap[\eta]=\Delta$. Moreover, if $[\rho]$ is any congruence on $S$ then

$$
[\rho]=([\rho] \vee \mathscr{H}) \cap([\rho] \vee[\eta]) .
$$

Proof. Let $(x, y) \in[\eta] \cap \mathscr{H} . S$ is a band of groups. Hence there is an idempotent $e \in H_{x}=H_{y}$. Since $(x, y) \in[\eta]$ there are $f, g \in E(S)$ such that $y=f x g$. Then

$$
y=e y e=e(f x g) e=e(f(\text { exe }) g) e=(e f e) x(\text { ege })=e x e=x .
$$


Thus $\mathscr{H} \cap[\eta]=\Delta$.

It is easily seen that if $[\rho]$ is any congruence on $S$ then $(x, y) \in[\rho] \vee \mathscr{H}$ if and only if $\rho(x)$ and $\rho(y)$ are in the same $\mathscr{H}$-class of $\rho(S)$, and $(x, y) \in[\rho] \vee[\eta]$ if and only if $E(\rho(S)) \rho(x) E(\rho(S))=E(\rho(S)) \rho(y) E(\rho(S))$. It then follows that $[\rho]=([\rho] \vee \mathscr{H})$ $\cap([\rho] \vee[\eta])$.

Let $I$ denote the closed real interval with multiplication defined by $x y=\min \{x, y\}$. For a topological semigroup $S$ let $\mathscr{C}(S, I)$ be the set of closed congruences [ $\varphi$ ] on $S$ such that $\varphi(S)$ can be topologically and algebraically imbedded in $I$.

THEOREM 6.1. Let $S$ be a compact topological semigroup such that

(1) $S$ is a band of groups,

(2) $E(S)$ is a subsemigroup of $S$,

(3) $\mathscr{C}(S / \mathscr{D}, I)$ separates points.

Then $\mathscr{H} \mathscr{L}(S)$ separates points.

Proof. Let $x$ and $y$ be any pair of points of $S$. Since $S$ is a band of groups $\mathscr{H}$ is a closed congruence on $S$. Each subgroup of $S / \mathscr{H}$ is degenerate so $S / \mathscr{H}$ is an $H L$ semigroup. Hence $\mathscr{H} \in \mathscr{H} \mathscr{L}(S)$. Then if $(x, y) \notin \mathscr{H} x$ and $y$ would be separated by $\mathscr{H}$. Thus we may assume that $(x, y) \in \mathscr{H}$. This being the case $\eta(x) \neq \eta(y)$ where $[\eta]$ is the minimal normal semigroup congruence on $S$ as defined in Lemma 6.1. Thus we may assume that $S$ is normal. This implies that the map of $S$ onto $x S$ defined by $s \rightarrow \tau(x) s$ is a continuous homomorphism. Thus we may assume that $S$ has an identity element 1 and $1=\tau(x)$. Since $H_{1}$ is a group $y$ has an inverse with respect to 1 , call it $y^{-1}$. Let $z=x y^{-1}$. We will show that $z$ and 1 can be separated by a member of $\mathscr{H} \mathscr{L}(S)$. Then since this congruence restricted to $H_{1}$ will be a group congruence $x$ and $y$ will be separated by a member of $\mathscr{H} \mathscr{L}(S)$.

We consider two cases.

Case (i). Suppose that 1 is an isolated point in $E(S)$. Then $S \backslash H_{1}$ is a closed ideal of $S . H_{1}$ is a compact topological group so there is a closed normal subgroup $K$ of $H_{1}$ such that $z \notin K$ and $H_{1} / K$ is a Lie group. Since $S$ is a normal semigroup the congruence on $H_{1}$ induced by $K$ can be extended to a congruence [ $\mu$ ] on $S$ by defining

$$
(s, t) \in[\mu] \text { if and only if } s K=t K \text {. }
$$

Let $\zeta$ be the natural map of $S$ onto the Rees-quotient $\mu(S) / \mu((E(S) \mid\{1\}) S)$. $\zeta(1)$ is the only nonzero idempotent of $\zeta(S)$ and $\zeta\left(H_{1}\right)$ is a Lie group. Hence $\zeta(S)$ must be an $H L$-semigroup and $[\zeta \circ \mu] \in \mathscr{H} \mathscr{L}$. $\zeta \circ \mu(z) \neq \zeta \circ \mu(1)$ so $[\zeta \circ \mu]$ is the desired congruence.

Case (ii). Suppose that 1 is not isolated in $E(S) . S$ is normal so $S / \mathscr{D}$ is iseomorphic with $E(S)$. Then since $\mathscr{C}(S / \mathscr{D}, I)$ separates points, $\mathscr{C}(E(S), I)$ separates points. This implies that there is a net $\left\{e_{\alpha} ; \alpha \in \Gamma\right\}$ of members of $E(S) \mid\{1\}$ which converges to 1 such that for each $\alpha \in \Gamma$ there is a homomorphism $\pi_{\alpha}$ of $E(S)$ onto a compact chain $E_{\alpha}$ having $e_{\alpha}$ as the zero of $\pi_{\alpha}^{-1}\left(\pi_{\alpha}(1)\right)$. For $s \in S$ we define $\xi_{\alpha}(s)$ 
to be the zero of $\pi_{\alpha}^{-1}\left(\pi_{\alpha}(\tau(s))\right)$. Since $S$ is a normal semigroup which is a union of groups by Theorem $3.1\left[\pi_{\alpha}\right]$ can be extended to a closed congruence $\left[\gamma_{\alpha}\right]$ on $S$. Because $S$ is compact and normal $\left[\gamma_{\alpha}\right]$ may be defined by

$$
(s, t) \in\left[\gamma_{\alpha}\right] \text { if and only if } \xi_{\alpha}(s) s=\xi_{\alpha}(t) t .
$$

If $e=\xi_{\alpha}(e)$ then $e$ satisfies condition (**) of Theorem 3.1. Hence $\gamma_{\alpha}$ restricted to $H_{e}$ is an isomorphism. Moreover, $E\left(\gamma_{\alpha}(S)\right)$ is isomorphic with $E_{\alpha}$. Since the net $\left\{e_{\alpha} ; \alpha \in \Gamma\right\}$ converges to 1 there is an $\alpha \in \Gamma$ such that $e_{\alpha} z \neq e_{\alpha}$. Thus $\gamma_{\alpha}(1) \neq \gamma_{\alpha}(z)$. If $\gamma_{\alpha}(1)$ is isolated in $E\left(\gamma_{\alpha}(S)\right)$ then by Case (i) there is a homomorphism of $\gamma_{\alpha}(S)$ onto an $H L$-semigroup which separates $\gamma_{\alpha}(1)$ and $\gamma_{\alpha}(z)$. The composition of this homomorphism with $\gamma_{\alpha}$ then gives rise to the desired congruence. Thus we may assume that $\gamma_{\alpha}(1)$ is not isolated in $E\left(\gamma_{\alpha}(S)\right)$. This implies that there is $e \in E\left(\gamma_{\alpha}(S)\right) \backslash\left\{\gamma_{\alpha}(1)\right\}$ such that $e \gamma_{\alpha}(z) \neq e$. Since $H_{e}$ is a compact topological group it has a closed normal subgroup $K_{e}$ such that $e \gamma_{\alpha}(z) \notin K_{e}$ and $H_{e} / K_{e}$ is a Lie group. Let $\rho$ denote the canonical homomorphism of $H_{e}$ onto $H_{e} / K_{e}$. On $\gamma_{\alpha}(S)$ we define a relation $[P]$ by

$$
\begin{aligned}
(s, t) \in[P] & \Leftrightarrow s, t \in e \gamma_{\alpha}(S), \quad \text { or } \\
& \Leftrightarrow e s e t \in H_{e}, \rho(e s)=\rho(e t), \quad \text { and } \\
& \Leftrightarrow(s, t) \in \mathscr{H} .
\end{aligned}
$$

Since the map $s \rightarrow e s$ is a homomorphism $[P]$ will be a closed congruence on $\gamma_{\alpha}(S)$. If $f \geqq e$ it follows that $\rho\left(e H_{f}\right)$ is a Lie group. This implies that $P\left(\gamma_{\alpha}(S)\right)$ is an $H L$ semigroup. Also $P \circ \gamma_{\alpha}(z) \neq P \circ \gamma_{\alpha}(1)$. Thus $P \circ \gamma_{\alpha}$ is the desired homomorphism.

$\mathscr{H}$ is a congruence on semigroups which satisfy the hypotheses of Theorem 6.1 and $\mathscr{H} \mathscr{L}$ is closed with respect to finite intersections so it follows that such semigroups have enough $\mathscr{H}$-classes separating homomorphisms onto $H L$-semigroups to separate points.

THEOREM 6.2. Let $S$ be a compact topological semigroup such that

(1) $H(S)$ is contained in the center of $S$.

(2) $\mathscr{C}(E(S), I)$ separates points.

Then $\mathscr{H} \mathscr{L}(S)$ separates points in $S$.

Proof. If $x \in S$ and $H_{x}$ is nondegenerate then since $S$ is compact there is a unique minimal idempotent $\omega(x)=e$ such that $H_{x}=x H_{e}$ (Lemma 3, [1]). Hence if $x \in S \backslash E(S) S$ then $H_{x}$ is degenerate. Then since $E(S) S$ is a closed ideal and $H(S)$ is contained in the center of $S$ it follows that $\mathscr{H} \cap(E(S) S \times E(S) S)$ is a congruence on $E(S) S$. Let $x, y \in E(S) S$ with $(x, y) \in \mathscr{H}$ and let $r \in S$. Then there is $g \in H_{\omega(x)}$ such that $g x=y$. Thus

$$
\operatorname{Sry}=\operatorname{Srg} x=\operatorname{Sgr} x=\operatorname{S\omega }(x) r x=\operatorname{Sr} \omega(x) x=\operatorname{Sr} x .
$$

From [16] it follows that $\mathscr{H}$ is a congruence on $S$. 
Now suppose that $x$ and $y$ are distinct elements of $S$. If $(x, y) \notin \mathscr{H}$ then $\mathscr{H} \in \mathscr{H} \mathscr{L}(S)$ and $\mathscr{H}$ separates $x$ and $y$. So we may assume that $(x, y) \in \mathscr{H}$. Then $x, y \in E(S) S$. Choose $g \in H_{\omega(x)}$ so that $g x=y$. From Theorem 6.1 there is $[\rho]$ $\in \mathscr{H} \mathscr{L}(H(S))$ such that $\rho(\omega(x)) \neq \rho(g)$. Let $K=\rho^{-1}(\rho(E(S)))$. We define a relation

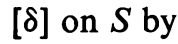

$$
(x, y) \in[\delta] \text { if and only if } x K=y K \text { or } x=y .
$$

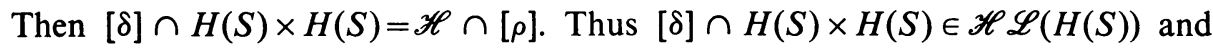
$[\delta] \in \mathscr{H} \mathscr{L}(S)$. Moreover $\delta(x) \neq \delta(y)$. So $[\delta]$ is the desired congruence.

\section{REFERENCES}

1. L. W. Anderson and R. P. Hunter, Homomorphisms and dimension, Math. Ann. 147 (1962), 248-268. MR 26 \#4324.

2. - On the infinite subsemigroups of certain compact semigroups (to appear).

3. J. T. Borrego, Adjunction semigroups, Bull. Austral. Math. Soc. 1 (1969), 47-58. MR 39 \#7021.

4. J. H. Carruth and C. E. Clark, Representations of certain compact semigroups by $H L$ semigroups, Trans. Amer. Math. Soc. 149 (1970), 327-337. MR 41 \#8563.

5. A. H. Clifford and G. B. Preston, The algebraic theory of semigroups. Vols. 1, 2, Math. Surveys, no. 7, Amer. Math. Soc., Providence, R. I., 1961, 1967. MR 24 \#A2627; MR 36 \#1558.

6. G. Gratzer, Lectures on lattice theory, Freeman, San Francisco, Calif., 1971.

7. J. M. Howie, Naturally ordered bands, Glasgow Math. J. 8 (1967), 55-58. MR 34 \#5726.

8. N. Kimura and M. Yamada, Note on idempotent semigroups. II, Proc. Japan Acad. 34 (1958), 110-112. MR 20 \#4603.

9. J. D. Lawson, Topological semilattices with small semilattices, J. London Math. Soc. (2) 1 (1969), 719-724. MR 40 \#6576.

10. M. Mislove, Four problems about compact semigroups, Dissertation, University of Tennessee, Knoxville, Tenn., 1969.

11. K. Numakura, Theorems on compact totally disconnected semigroups and lattices, Proc. Amer. Math. Soc. 8 (1957), 623-626. MR 19, 290.

12. A. R. Stralka, The Green equivalences and dimension in compact semigroups, Math. $Z$. 109 (1969), 169-176. MR 39 \#2903.

13. - The congruence extension property for compact topological lattices, Pacific J. Math. (to appear).

14. M. Yamada, Regular semigroups whose idempotents satisfy permutation identities, Pacific J. Math. 21 (1967), 371-392. MR 37 \#2887.

15. - On a regular semigroup in which the idempotents form a band, Pacific J. Math. 33 (1970), 261-272.

16. A. D. Wallace, Project $M O B$, Lecture Notes, University of Florida, Tallahassee, Fla., 1964, unpublished.

Department of Mathematics, University of California, Riverside, California 92502 